\title{
Intraoperative pleural lavage cytology is an independent prognostic indicator for staging non-small cell lung cancer
}

\author{
Eric Lim, MRCS ${ }^{a}$ \\ Ayyaz Ali, MRCS ${ }^{a}$ \\ Panagiotis Theodorou, $\mathrm{MD}^{\mathrm{a}}$ \\ Andrew G. Nicholson, FRCPath ${ }^{b}$ \\ George Ladas, FETCS ${ }^{a}$ \\ Peter Goldstraw, FRCS ${ }^{\mathrm{a}}$
}

See related editorial on page 947.
From the Departments of Thoracic Surgery $^{\mathrm{a}}$ and Histopathology, ${ }^{\mathrm{b}}$ Royal Brompton Hospital, London, United Kingdom.

Received for publication May 26, 2003; revisions received Sept 8, 2003; accepted for publication Oct 2, 2003.

Address for reprints: Peter Goldstraw, Department of Thoracic Surgery, Royal Brompton Hospital, Sydney St, London SW3 6NP, United Kingdom (E-mail: p.goldstraw@rbh.nthames.nhs.uk).

J Thorac Cardiovasc Surg 2004;127:1113-8

$0022-5223 / \$ 30.00$

Copyright $(92004$ by The American Association for Thoracic Surgery

doi:10.1016/j.jtcvs.2003.10.025
Objectives: For patients undergoing lung resection for cancer, macroscopic evidence of metastasis is clearly associated with adverse prognosis. However, less is known about the significance of tumor cells detected by using tests such as pleural lavage cytology. To ascertain the frequency and quantify the effect of this finding on survival, we performed a prospective study of intraoperative pleural lavage cytology.

Methods: Pleural lavage cytology consisted of cytologic analysis of $100 \mathrm{~mL}$ of saline irrigated over the lung surface immediately after thoracotomy. Patients were excluded if they had an existing effusion, extreme adhesions, or lateral chest wall invasion or if resection was not performed. Survival was calculated by means of Kaplan-Meier analysis and compared by using log-rank tests. Cox regression was used to ascertain independent predictors of prognosis.

Results: From 1995 through 2003, we performed pleural lavage cytology on 292 patients undergoing thoracotomy for lung cancer. The mean age was 64 (SD, 10) years, and $196(67 \%)$ patients were men. Of 292 samples, $13(4.5 \%)$ showed evidence of malignant cells. The median time to follow-up was 15 months (interquartile range, 1-40 months), with a median survival of 49 months for patients with negative pleural lavage cytology results and 13 months for patients with positive pleural lavage cytology results $(P=.002)$. Univariate prognostic predictors were positive pleural lavage cytology status $(P=.03)$, stage $(P=.03)$, adenocarcinoma $(P=.06)$, and parietal pleural involvement $(P=.01)$. In the final multivariate model only positive pleural lavage cytology status $(P=.006)$ and stage $(P=.03)$ remained significant.

Conclusions: Intraoperative pleural lavage cytology is a simple addition to intrathoracic staging and an independent predictor of prognosis. Positive results potentially affect survival by upstaging patients to stage IIIB or greater.

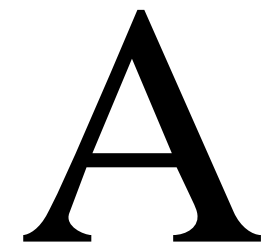

ccurate and reproducible staging is the fundamental basis for patient management, evaluation of research, and communication in the treatment of lung cancer. ${ }^{1}$ Investigating for local, lymphatic, and hematogenous invasion is part of the routine evaluation before lung resection. Although these staging methods tend to concentrate on macroscopic evidence of disseminated disease, advances in cytochemistry, immunohistochemistry, and polymerase chain reaction techniques facilitate detection of micrometastatic disease. ${ }^{2}$ In patients without evidence of metastasis, isolated tumor cells have been detected in the bone marrow and lymph nodes, even though conventional stains have failed to detect such disease. $^{3,4}$ 
Because these findings are becoming increasingly clinically relevant, thoracic surgeons are now evaluating the significance of tumor cells detected on washings of the pleural cavity. The contribution to staging and the utility to influence subsequent patient management are not clearly defined.

In an attempt to ascertain the frequency of tumor cells on pleural washings and to characterize the effect on prognosis, we performed a prospective study of pleural lavage cytology (PLC) in patients who had thoracotomy and lung resection for non-small cell lung cancer (NSCLC) at our institution.

\section{Methods}

This study was conducted from 1995 through 2003, including all patients with known or suspected NSCLC who had a thoracotomy for intended surgical resection. Patients with a confirmed diagnosis of NSCLC formed the population for this study, and we did not include any patients with benign disease. We excluded patients who had an existing effusion at the time of the operation (defined as visible evidence of fluid in the pleural cavity), extreme adhesions, or chest wall invasion or in whom resection was not performed.

\section{Preparation of the Sample}

Immediately after thoracotomy but before any manipulation of the tumor, $100 \mathrm{~mL}$ of normal saline was instilled into the pleural space through a wide-bore catheter. The fluid was irrigated over the visceral and parietal pleura, but forceful irrigation over the tumor was avoided. The sample was then aspirated and placed in a sterile container with $1 \mathrm{~mL}$ of $20 \%$ sodium citrate. The sample was mixed by means of repeated inversion. Isolation of cells from erythrocytes was achieved by using a centrifugal sedimentation system. Two 16-mL centrifuged tubes were prepared by layering $12 \mathrm{~mL}$ of sample over $3 \mathrm{~mL}$ of Lymphoprep medium (Nycomed Pharma AS) and centrifuging at $2000 \mathrm{rpm}$ for 20 minutes. Cells were harvested from the sample-medium interface with a Pasteur pipette and resuspended in $2 \mathrm{~mL}$ of supernatant. Four cytologic slides of this fraction were prepared by using a Cyto-Tek centrifuge (Bayer Diagnostics), subsequently fixed in formal acetic alcohol, and stained with the Papanicolaou technique. The slides were then screened by a pathologist (AGN) for the presence of malignant cells.

\section{Data Acquisition}

Individual patient data were collated from a prospective histopathology database. Survival status was determined from the date of last follow-up in a hospital outpatient or general practitioner's clinic. Mortality status was documented from patient records and the NHS strategic tracing service.

\section{Statistical Analysis}

Patients were grouped according to the results of intraoperative PLC. Categoric data are presented as frequency (in percentages) and continuous data as means with SDs or medians with interquartile ranges. Comparisons of categoric data between the 2 groups were made by using $\chi^{2}$ or Fisher exact tests. Continuous data were compared by using 2-tailed $t$ tests or Mann-Whitney tests as appropriate to the distribution of the data.

Actuarial survival was estimated by using the Kaplan-Meier method and compared with the log-rank test. Cox proportional hazards regression was used to ascertain the individual contribution of factors associated with survival and to compare the riskadjusted survival between the 2 groups. The criterion for variable retention was a $P$ value of less than .1.

\section{Results}

From January 1, 1995, to January 1, 2003, a total of 292 PLC samples were received from patients who fulfilled the inclusion criteria and underwent surgical resection for NSCLC.

The mean age of the cohort was $64(\mathrm{SD}, 10)$ years, and $196(67 \%)$ of the patients were men. Of 292 samples, 13 (4.5\%) had evidence of malignant cells. Patients with positive PLC results were well matched for age and sex. However, more underwent pneumonectomy and had a higher stage compared with patients who had negative PLC results. In our series all patients with stage IV disease had M1 status by virtue of a second tumor in a different lobe. The baseline characteristics and stage are summarized in Tables 1 and 2, respectively.

The median time to follow-up was 15 months (interquartile range, 1-40 months), with a median survival of 49 (SE, 2.81) months for patients with negative PLC results and 13 (SE, 8.9) months for patients with positive PLC results $(P=$ .002 , Figure 1).

The univariate prognostic predictors were positive PLC status $(P=.03)$, stage $(P=.03)$, adenocarcinoma $(P=$ $.06)$, and parietal pleural involvement $(P=.01)$, as summarized in Table 3. During statistical modeling, PLC status $(P=.002)$ as a prognostic predictor was independent of $\mathrm{N}$ stage $(P<.001)$ and $\mathrm{T}$ stage $(P=.9)$. In the final multivariate model, only positive PLC status $(P=.006)$ and overall stage $(P=.03)$ were retained as independent prognostic predictors (Table 4 and Figure 2).

A subanalysis of the 12 patients with positive PLC results with stage IB to IIIB disease (after excluding one patient with stage IV disease) compared with the 17 patients with stage IIIB disease in the group with negative PLC results suggested poorer survival when PLC results were positive $(P=.06$, Figure 3$)$.

\section{Discussion}

How common is intracoelomic disease in patients who undergo lung resection without evidence of pleural effusion? In our series the proportion was relatively low (4.5\%). However, reports in the literature range between $9 \%$ and $38 \%$. The proportion of positive lavage results varies not only with the patient population but also with the technique of acquiring the sample. The volume of lavage fluid used to 
TABLE 1. Baseline characteristics, operation extent, and cell type

\begin{tabular}{|c|c|c|c|}
\hline & PLC negative & PLC positive & $P$ value \\
\hline No. & 279 & 13 & \\
\hline Age, y (SD) & $64(11)$ & $63(7)$ & .84 \\
\hline Male, n (\%) & $188(67)$ & $8(62)$ & .66 \\
\hline \multicolumn{4}{|l|}{ Operation } \\
\hline Pneumonectomy, n (\%) & $57(20)$ & $5(38)$ & \\
\hline Lobectomy, n (\%) & $193(69)$ & $5(38)$ & \\
\hline Bilobectomy, n (\%) & $15(5)$ & $1(8)$ & .03 \\
\hline Segmentectomy, n (\%) & $7(2)$ & $0(0)$ & \\
\hline Nonanatomic resection, n (\%) & $7(3)$ & $2(15)$ & \\
\hline \multicolumn{4}{|l|}{ Histology } \\
\hline Squamous, n (\%) & $114(41)$ & $2(15)$ & \\
\hline Adenocarcinoma, n (\%) & $118(42)$ & $10(77)$ & \\
\hline Large cell, $\mathrm{n}(\%)$ & $23(8)$ & $0(0)$ & .12 \\
\hline Mixed, n (\%) & $14(5)$ & $1(8)$ & \\
\hline Other, $\mathrm{n}(\%)$ & $10(4)$ & $0(0)$ & \\
\hline Visceral pleura involvement, $\mathrm{n}(\%)$ & $87(31)$ & $7(54)$ & .08 \\
\hline Parietal pleura involvement, $\mathrm{n}(\%)$ & 6 & 1 & .21 \\
\hline
\end{tabular}

acquire a sample ranges from 50 to $1500 \mathrm{~mL}$, and different carrier solutions, such as saline and Ringer's lactate have been used. ${ }^{3,5-9}$ Generally, most samples have been acquired immediately after thoracotomy and before surgical intervention. ${ }^{3,6,8}$ However, some have performed PLC after surgical intervention, ${ }^{10}$ and others have performed PLC both before and after lung resection. ${ }^{5,7}$ Despite the inconsistencies in volume, carrier solutions, timing of collection, and processing, one finding has been consistent: a uniformly poor prognosis in patients with positive results.

\section{Role for PLC in Staging}

Although some studies have identified positive PLC results as an independent predictor of prognosis, ${ }^{6}$ not all studies that report a poorer survival were able to confirm whether this was independent of stage. ${ }^{3}$ This problem stems from small numbers of patients with positive PLC results and the variable effect on survival in each series. Our study supports positive PLC results as a prognostic indicator that is independent of overall stage and, in particular, nodal status. Where and how PLC could be implemented into conventional lung cancer staging is still unclear. It is recommended that the cytologic results of pleural and peritoneal washings be considered separate to the classification of isolated tumor cells and micrometastasis. In addition, identification of patients with positive PLC results requires the suffix of (cy+) if this is to be the basis of classification of this subset to $\mathrm{T} 4$ disease (ie, T4 [cy+]). ${ }^{11}$

In our series patients with positive PLC results had equivalent or possibly poorer survival to that of patients with stage IIIB disease $(P=.06)$. This is despite having $83 \%(10 / 12)$ of such patients with stage IB to IIIA disease. Clearly, positive PLC results have the effect of upstaging patients to at least $\mathrm{T} 4$ status, a finding with prognostic
TABLE 2. Staging characteristics

\begin{tabular}{lccc}
\hline & PLC negative & PLC positive & $P$ value \\
\hline No. & 279 & 13 & \\
Stage & & & \\
IA, n (\%) & $51(18)$ & $0(0)$ & \\
IB, n (\%) & $108(39)$ & $3(23)$ & \\
IIA, n (\%) & $12(4)$ & $1(8)$ & .01 \\
IIB, n (\%) & $53(19)$ & $1(8)$ & \\
IIIA, n (\%) & $35(13)$ & $5(38)$ & \\
IIIB, n (\%) & $17(6)$ & $2(15)$ & \\
IV, n (\%) & $3(1)$ & $1(8)$ & \\
T category & & & \\
T1, n (\%) & $74(27)$ & $3(23)$ & \\
T2, n (\%) & $181(65)$ & $8(62)$ & \\
T3, n (\%) & $8(3)$ & $0(0)$ & \\
T4, n (\%) & $16(6)$ & $2(15)$ & \\
N category & & & \\
N0, n (\%) & $197(71)$ & $8(62)$ & \\
N1, n (\%) & $47(17)$ & $1(8)$ & \\
N2, n (\%) & $35(13)$ & $4(31)$ & \\
\hline
\end{tabular}

implications similar to those of a malignant effusion. Although we did not have a group of patients with stage IV disease to ascertain whether survival was comparable, the median survival of 13 months is similar to that of patients with stage IV disease reported by Naruke and colleagues. ${ }^{12}$

\section{A Unique Problem}

Currently, patients with stage IIIB and IV disease are generally (with few exceptions) considered to have inoperable disease, ${ }^{13}$ and surgeons can justifiably choose not to proceed to lung resection when faced with unexpected stage IIIB or IV disease in the operating theater. However, patients with positive PLC results belong to a small subgroup 


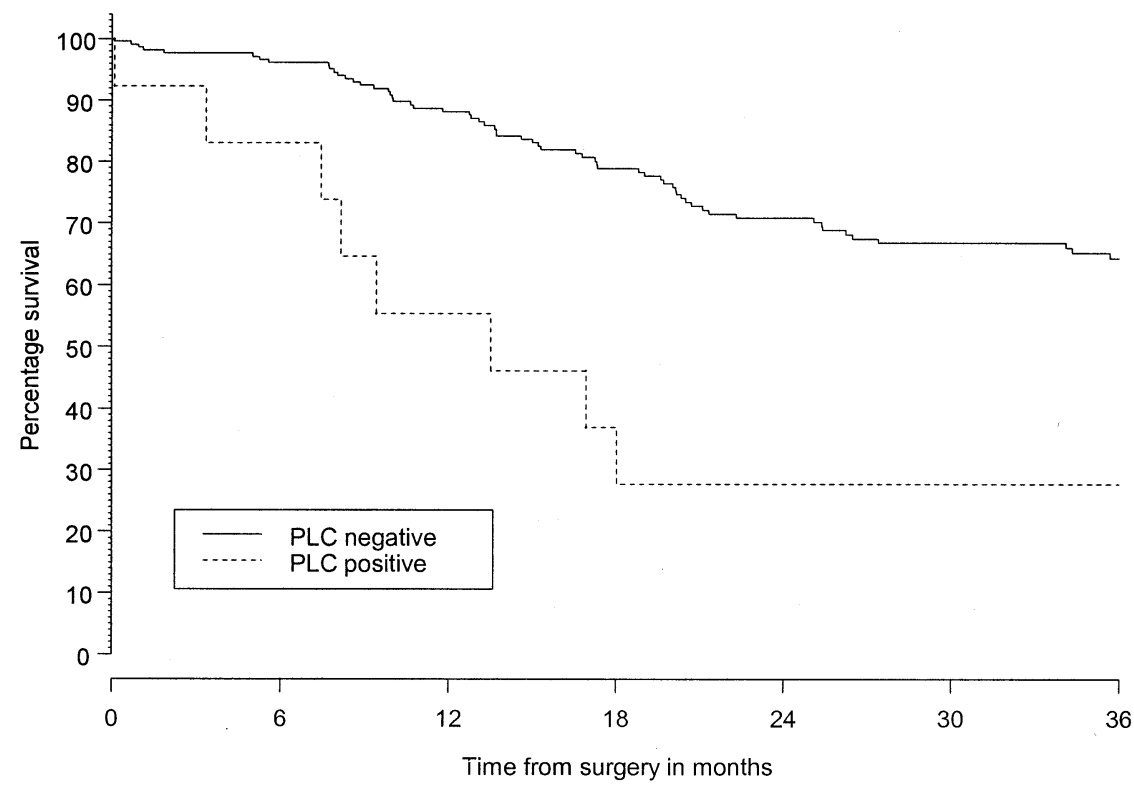

Figure 1. Overall survival by PLC status.

TABLE 3. Univariate predictors of survival

\begin{tabular}{lcc}
\hline Variable & Odds ratio & P value \\
\hline PLC & 2.45 & .03 \\
Stage & & $(.03)^{*}$ \\
I & 1.00 & \\
II & 1.16 & .29 \\
III & 1.23 & .04 \\
IV & 1.01 & .95 \\
Age (per additional yr) & 1.02 & .12 \\
Female sex & 0.87 & .28 \\
Extent of resection & & \\
Pneumonectomy & 1.00 & \\
Lobectomy & 0.82 & .17 \\
Bilobectomy & 0.91 & .58 \\
Segmentectomy & 1.13 & .63 \\
Nonanatomic resection & 0.86 & .47 \\
Histology & & \\
Squamous & 1.00 & \\
Adenocarcinoma & 1.31 & .06 \\
Large cell & 1.18 & .19 \\
Mixed & 0.99 & .92 \\
Other & 0.35 & .56 \\
Maximum size (per additional mm) & 0.99 & .52 \\
Visceral pleura involvement & 0.88 & .62 \\
Parietal pleura involvement & 10.33 & .01 \\
\end{tabular}

*The overall contribution of stage was assessed by using the likelihood method.

with disease that is not detectable by means of conventional staging methods and who might have surgically resectable disease with a pathologic stage as early as IB (in our series). Because we are unlikely to develop an infrastructure that is quick enough to provide us the necessary information in-
TABLE 4. Multivariate predictors of survival

\begin{tabular}{lcc}
\hline Variable & Odds ratio & $\boldsymbol{P}$ value \\
\hline $\begin{array}{l}\text { Positive PLC results } \\
\text { Stage }\end{array}$ & 2.52 & .006 \\
I & 1.00 & $(.03)^{*}$ \\
II & 1.17 & .21 \\
III & 1.23 & .01 \\
IV & 1.07 & .80 \\
\hline
\end{tabular}

*The overall contribution of stage was assessed by using the likelihood method.

traoperatively, the results of PLC are not available until after the operation, compounding the problem of the management of these patients. Even if we did use preoperative video-assisted thoracoscopy to obtain lavage fluid for PLC, the cost and potential morbidity for a detection rate of $4.5 \%$ would far outweigh the clinical utility of this investigation, especially when we do not as yet have a clear management plan for patients with positive results. With our present knowledge, it would not be appropriate to turn down patients with potentially resectable disease in the absence of further confirmatory studies.

\section{Clinical Implications}

Clearly the challenge is to define the optimal management strategy for this small but important subset of patients. A recent study of this subgroup by Ichinose and associates ${ }^{14}$ suggested reduced carcinomatous pleuritis in patients with positive PLC results who had hypotonic cisplatin infused into the pleural space after lung resection. However, the study terminated prematurely before the effects on recur- 


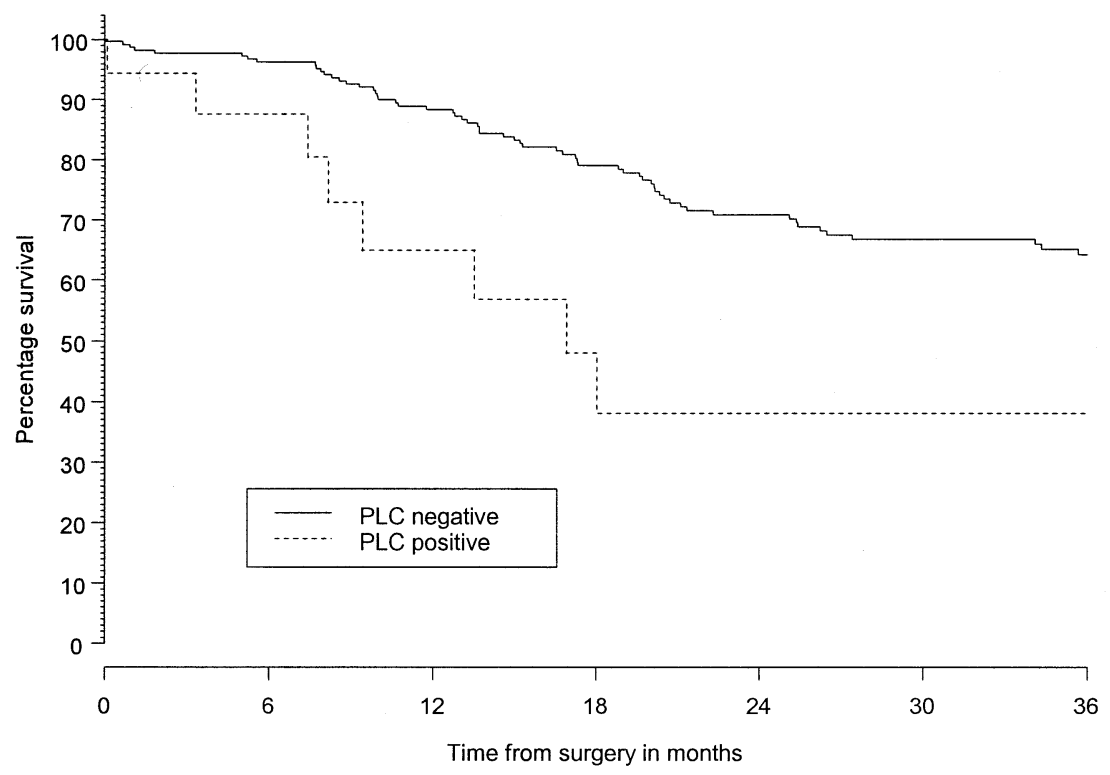

Figure 2. Survival by PLC status adjusted for stage.

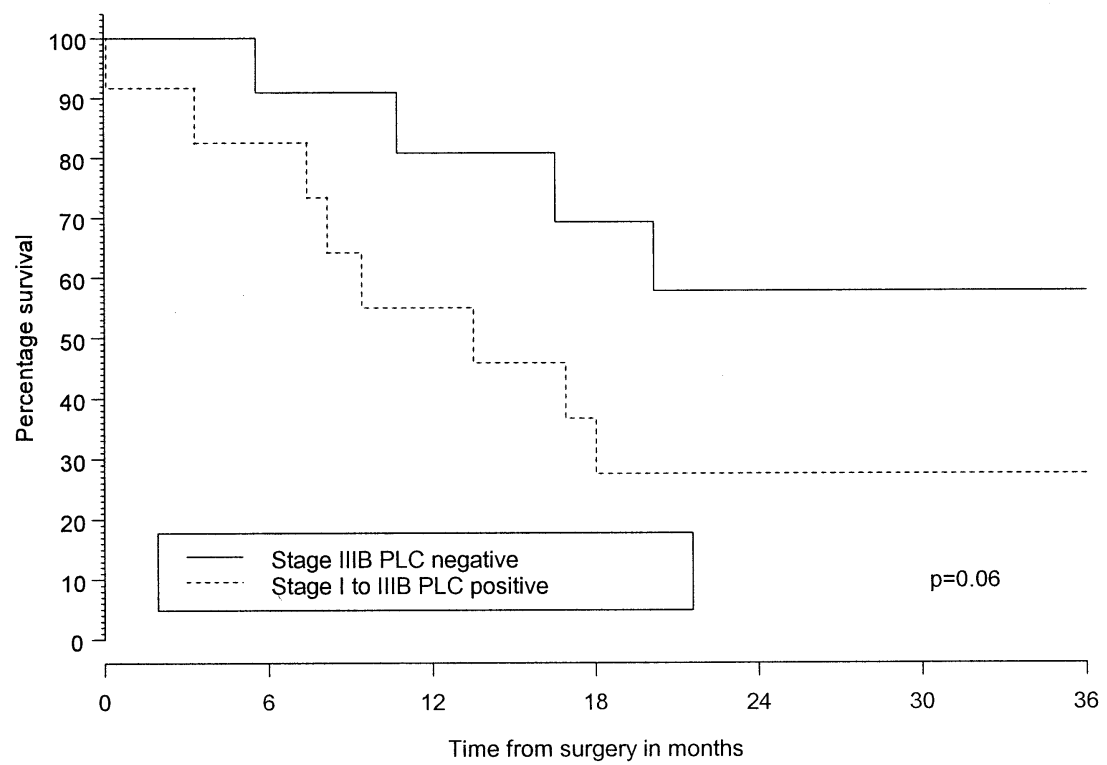

Figure 3. Survival comparing patients with stage IIIB disease with all patients with positive PLC results.

rence or mortality could be assessed. Realistically, until we can accurately and consistently identify patients in this subgroup, it would be difficult to evaluate the benefits of any adjuvant therapy. Moreover, this is based on the assumption that positive PLC results are an indicator of pleural extravasation of the primary tumor. It is equally conceivable that positive PLC results occur as a result of increasing metastatic potential and widespread microscopic disease detected on pleural washings.

\section{Potential Limitations}

Like many other studies on this topic, the numbers of patients with positive PLC results were small, limiting the ability to make robust conclusions. However, our data are clearly consistent with those of other reports as an adverse prognostic indicator. Because of sample size constraints, we were unable to ascertain with certainty whether visceral pleural involvement was associated with positive PLC results, and we did not have information on disease recurrence. 


\section{Future Direction}

PLC is a relatively inexpensive (approximate cost is $£ 40$, US \$65.45) and simple technique that identifies an important subset of surgical candidates with poor prognosis. However, standardization of sample acquisition and preparation is essential for widespread adoption and interpretation of future studies. Although numerous studies have been published on this topic, further studies or individual patient data meta-analyses of published trials are required to facilitate the accurate redefinition of a stage to this subgroup of patients.

\section{Conclusions}

Intraoperative PLC is a simple and inexpensive technique that identifies surgical patients with micrometastasis otherwise undetectable by means of conventional staging methods. The information provided by PLC conveys important additional prognostic information and potentially affects survival by upstaging patients to stage IIIB or greater. Standardization of sample acquisition and further studies are required to define the exact stage of patients with positive PLC results before it becomes widely adopted for staging. Further information on relapse patterns is needed before the prognostic information obtained can be used to assess the effect of adjuvant therapy.

We gratefully acknowledge the contribution of Christopher Jackson, $\mathrm{PhD}$, for his assistance with the statistical analysis for this study.

\section{References}

1. Mountain CF. Revisions in the International System for Staging Lung Cancer. Chest. 1997;111:1710-7.

2. Jiao X, Krasna MJ. Clinical significance of micrometastasis in lung and esophageal cancer: a new paradigm in thoracic oncology. Ann Thorac Surg. 2002;74:278-84.

3. Hillerdal G, Dernevik L, Almgren SO, Kling PA, Gustafsson G. Prognostic value of malignant cells in pleural lavage at thoracotomy for bronchial carcinoma. Lung Cancer. 1998;21:47-52.

4. Gu CD, Osaki T, Oyama T, Inoue M, Kodate M, Dobashi K, et al. Detection of micrometastatic tumor cells in pN0 lymph nodes of patients with completely resected nonsmall cell lung cancer: impact on recurrence and Survival. Ann Surg. 2002;235:133-9.

5. Okumura M, Ohshima S, Kotake Y, Morino H, Kikui M, Yasumitsu T. Intraoperative pleural lavage cytology in lung cancer patients. Ann Thorac Surg. 1991;51:599-604.

6. Kondo H, Asamura H, Suemasu K, Goya T, Tsuchiya R, Naruke T, et al. Prognostic significance of pleural lavage cytology immediately after thoracotomy in patients with lung cancer. $J$ Thorac Cardiovasc Surg. 1993;106:1092-7.

7. Kjellberg SI, Dresler CM, Goldberg M. Pleural cytologies in lung cancer without pleural effusions. Ann Thorac Surg. 1997;64:941-4.

8. Buhr J, Berghauser KH, Gonner S, Kelm C, Burkhardt EA, Padberg WM. The prognostic significance of tumor cell detection in intraoperative pleural lavage and lung tissue cultures for patients with lung cancer. J Thorac Cardiovasc Surg. 1997;113:683-90.

9. Dresler CM, Fratelli C, Babb J. Prognostic value of positive pleural lavage in patients with lung cancer resection. Ann Thorac Surg. 1999; 67:1435-9.

10. Kotoulas C, Lazopoulos G, Karaiskos T, Tomos P, Konstantinou M, Papamichalis G, et al. Prognostic significance of pleural lavage cytology after resection for non-small cell lung cancer. Eur J Cardiothorac Surg. 2001;20:330-4.

11. Hermanek P, Hutter RV, Sobin LH, Wittekind C. International Union Against Cancer. Classification of isolated tumor cells and micrometastasis. Cancer. 1999;86:2668-73.

12. Naruke T, Tsuchiya R, Kondo H, Asamura H. Prognosis and survival after resection for bronchogenic carcinoma based on the 1997 TNMstaging classification: the Japanese experience. Ann Thorac Surg. 2001;71:1759-64.

13. Mountain CF. Staging classification of lung cancer. A critical evaluation. Clin Chest Med. 2002;23:103-21.

14. Ichinose Y, Tsuchiya R, Koike T, Yasumitsu T, Nakamura K, Tada H, et al. A prematurely terminated phase III trial of intraoperative intrapleural hypotonic cisplatin treatment in patients with resected nonsmall cell lung cancer with positive pleural lavage cytology: the incidence of carcinomatous pleuritis after surgical intervention. $J$ Thorac Cardiovasc Surg. 2002;123:695-9. 